\title{
Continuous control of local magnetic moment by applied electric field in multiferroics $\mathrm{Ba}_{2} \mathrm{CoGe}_{2} \mathrm{O}_{7}$
}

\author{
Minoru Soda, ${ }^{1}$ Shohei Hayashida, ${ }^{1}$ Bertrand Roessli, ${ }^{2}$ Martin Månsson, ${ }^{2,3}$ Jonathan S. White, ${ }^{2}$ Masashige Matsumoto, ${ }^{4}$ \\ Ryousuke Shiina, ${ }^{5}$ and Takatsugu Masuda ${ }^{1}$ \\ ${ }^{1}$ Neutron Science Laboratory, Institute for Solid State Physics, University of Tokyo, Tokai, Ibaraki 319-1106, Japan \\ ${ }^{2}$ Laboratory for Neutron Scattering and Imaging, Paul Scherrer Institut, CH-5232 Villigen PSI, Switzerland \\ ${ }^{3}$ Department of Materials and Nanophysics, KTH Royal Institute of Technology, SE-164 40 Kista, Sweden \\ ${ }^{4}$ Department of Physics, Shizuoka University, Shizuoka 422-8529, Japan \\ ${ }^{5}$ Faculty of Science, University of the Ryukyus, Nishihara, Okinawa 903-0213, Japan
}

(Received 12 July 2016; published 16 September 2016)

\begin{abstract}
$\mathrm{Ba}_{2} \mathrm{CoGe}_{2} \mathrm{O}_{7}$ exhibits a collinear-antiferromagnetic structure with the easy axis along $\langle 100\rangle$ directions and an antiferroelectric order with the polarization axis along the [001] direction. By applying the electric field the magnetic moment rotates from $\langle 100\rangle$ to [110] directions and, simultaneously, the antiferroelectric state changes to the ferroelectric state gradually. This magnetoelectric effect, i.e., continuous control of the local magnetic moment by the electric field, is quantitatively explained by the Hamiltonian including the dielectric energy.
\end{abstract}

DOI: 10.1103/PhysRevB.94.094418

\section{INTRODUCTION}

Strong coupling between magnetism and dielectricity has attracted great attention [1,2]. In some materials, an electric polarization $P$ is controlled by the nonconjugate quantity, magnetic field $H$, and so is a magnetization $M$ by the electric field $E[1,3]$. This is known as the magnetoelectric (ME) effect. The ME effect was first discovered in $\mathrm{Cr}_{2} \mathrm{O}_{3}$ and the linear ME effect, in which $P$ and $M$ are in proportion to $H$ and $E$, respectively, has been studied [4,5]. In the linear $\mathrm{ME}$ effect the coupling constant between $P$ and $H$, or $M$ and $E$, is small $[6,7]$. In contrast in multiferroics where an electric polarization and a magnetic order coexist [3], the ME effect is nonlinear and strongly enhanced. The origin of the multiferroic property has been studied both experimentally [8-10] and theoretically [11-14].

Here we focus on the control of the magnetism by the electric field in the multiferroics. The electric control of the magnetism without an energy loss is important from an application point of view. Nonlinear ME effects, such as the reversal of the ferromagnetic component with the electric field $[15,16]$, the electric control of the direction of the magnetization $[17,18]$, and the electric control of the spin chirality [19-21], were reported so far. In these ME effects, the magnetic domain is aligned by the electric field [22,23], and the dielectric energy of the system does not change. Recently the ME effect with the change of the anisotropy energy has been discussed in $\mathrm{Cu}_{2} \mathrm{OSeO}_{3}$ where the nanoscale skyrmion lattice composed of many magnetic moments is rotated by $E$ and oscillating $H$ [24]. The next challenge is to find a ME effect where the local magnetic moment changes continuously with the dielectric energy, and we target the multiferroic system $\mathrm{Ba}_{2} \mathrm{CoGe}_{2} \mathrm{O}_{7}$ having the collinear-magnetic structure.

The $\mathrm{Ba}_{2} \mathrm{CoGe}_{2} \mathrm{O}_{7}$ has a two-dimensional layer composed of the network of $\mathrm{CoO}_{4}$ and $\mathrm{GeO}_{4}$ tetrahedra as shown in Fig. 1(a). $\mathrm{Co}^{2+}$ ions have spin $S=3 / 2$ and form a square lattice. An antiferromagnetic ordering with a collinearmagnetic structure occurs at $T_{\mathrm{N}}=6.7 \mathrm{~K}[25,26]$. Below $T_{\mathrm{N}}$ the system has a ferroelectricity induced by $H$ and the electric polarization continuously changes with increasing $H[27,28]$. The magnitude and direction of the electric polarization depend on the relationship between the local Co spin and the $\mathrm{O}$ ions in $\mathrm{CoO}_{4}$ tetrahedra. This is explained by the spin-dependent $d$-p hybridization mechanism

$$
\boldsymbol{p} \propto \sum_{i}\left(\boldsymbol{S} \cdot \boldsymbol{e}_{i}\right)^{2} \boldsymbol{e}_{i},
$$

where $\boldsymbol{e}_{i}$ is the vector connecting the Co and the $i$ th $\mathrm{O}$ ions $[13,14]$.

The effective Hamiltonian for the spin states of $S=3 / 2$ in $\mathrm{Ba}_{2} \mathrm{CoGe}_{2} \mathrm{O}_{7}$ is composed of the conventional bilinear spin interaction $\mathcal{H}_{x x z}$, the single ion anisotropy $\mathcal{H}_{D}$, a Dzyaloshinskii-Moriya interaction $\mathcal{H}_{D M}$, and a spin-nematic interaction $\mathcal{H}_{p}$ [29]. The first two terms stabilize a collinear antiferromagnetic structure with reduced spins, leaving the spin directions unfixed. The spin-nematic interaction, which is equivalent to an interaction of local electric polarizations, is the only source of in-plane anisotropy, and it is given by

$$
\mathcal{H}_{p}=-J_{p} \sum_{i, j} p_{Z}^{(i)} p_{Z}^{(j)}=-J_{p}^{\mathrm{eff}} \sum_{i, j} O_{X Y}^{(i)} O_{X Y}^{(j)},
$$

where $O_{X Y}=S^{X} S^{Y}+S^{Y} S^{X}$ is the spin-nematic operator [30,31]. The local electric polarization of the $\mathrm{CoO}_{4}$ tetrahedron along $Z$ direction, corresponding to the crystallographic $c$ axis, is $p_{Z}$, and $p_{Z}$ is equal to $K_{c} O_{X Y}$ with constant $K_{c}$. The negative and positive values of an effective coupling strength $J_{p}^{\text {eff }}$ mean antiferroelectric and ferroelectric interactions, respectively. The antiferroelectric state relates to the magnetic structure with the easy axis along $\langle 100\rangle$ as shown in Fig. 1(b) and the ferroelectric state relates to the magnetic structure with the easy axis along $\langle 110\rangle$ as shown in Fig. 1(e). Our previous study showed that the former case is realized with the spin-nematic interaction of $J_{p}^{\text {eff }}=-0.198 \mu \mathrm{eV}$ [29].

What will happen to the local magnetic moment if the electric field is applied? In the case that $E$ is applied along the $c$ axis in the antiferroelectric state, the local electric polarization of $\mathrm{CoO}_{4}$ changes with its direction fixed to the $E$. The direction of magnetic moment simultaneously changes because it is determined by the direction and magnitude of the local polarization in considering Eq. (1). This means that the ground state in Fig. 1(b) changes to the $E$-induced state in 

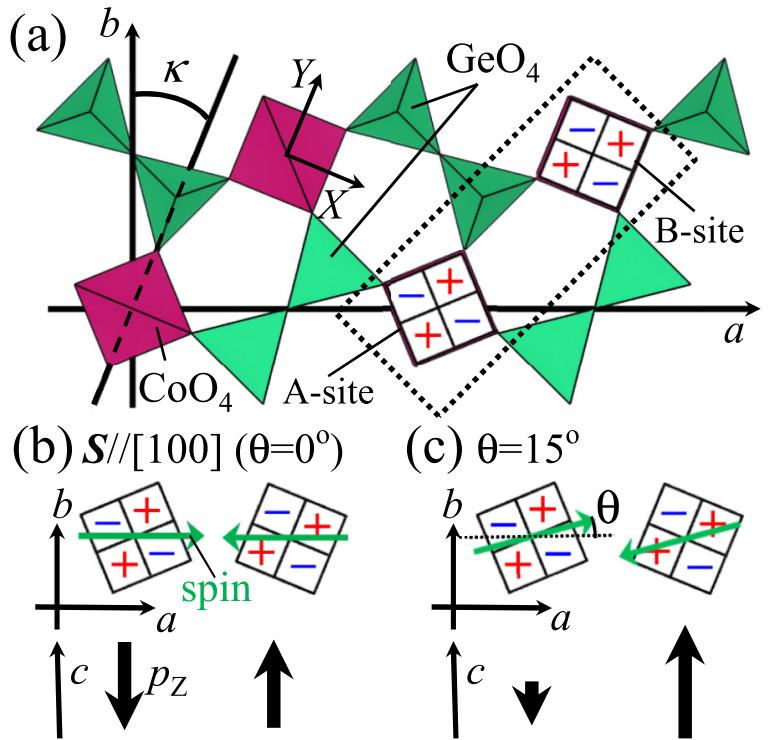

(d) $\theta=30^{\circ}$
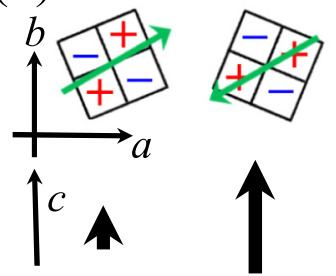

(c) $\theta=15^{\circ}$

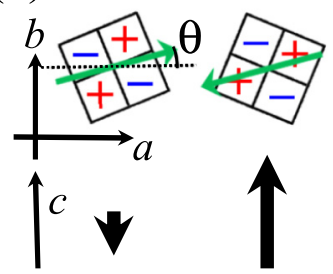

(e) $S / /[110]\left(\theta=45^{\circ}\right)$

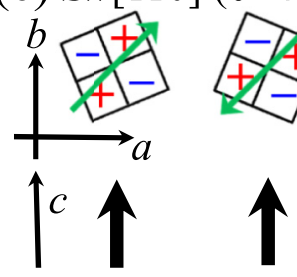

FIG. 1. (a) Two-dimensional lattice composed of $\mathrm{CoO}_{4}$ and $\mathrm{GeO}_{4}$ tetrahedra in $\mathrm{Ba}_{2} \mathrm{CoGe}_{2} \mathrm{O}_{7}$. Plus and minus signs in $\mathrm{CoO}_{4}$ show the relationship between the sign of $\left\langle O_{X Y}\right\rangle$ and the magnetic moment direction. (b)-(e) Schematic relationships between the directions of the magnetic moment and the local electric polarization of $\mathrm{CoO}_{4}$ tetrahedra. The ground state has the antiferroelectric state and the magnetic structure with the easy axis along $\langle 100\rangle$. The ferroelectric state relates to the magnetic structure with easy axis along $\langle 110\rangle$.

Fig. 1(e). In the present study we investigate the $E$ dependence of the magnetic structure of $\mathrm{Ba}_{2} \mathrm{CoGe}_{2} \mathrm{O}_{7}$ to clarify the electric control of the local magnetic moment through the spin-nematic interaction. In this paper we demonstrate that the magnetic moment rotates continuously in the $c$ plane with increasing $E$. The rotation of the magnetic moment is induced by the dielectric-energy change.

\section{EXPERIMENTAL DETAILS}

The $\mathrm{Ba}_{2} \mathrm{CoGe}_{2} \mathrm{O}_{7}$ single crystal was grown by the floatingzone method. Polarized-neutron measurement was carried out using the cold-neutron triple-axis spectrometer TASP installed at SINQ/PSI, Switzerland, to measure the magnetic component separately from the nuclear one [32]. The tetragonal unit cell where the lattice constants are $a=b=8.410 \AA$ and $c=5.537 \AA$ was used, and the scattering plane was the $a-b$ plane. For the single crystal with $2.5 \mathrm{~mm}$ thickness, aluminum electrodes were deposited onto the faces of (001) to apply $E$. Maximum voltage in the used equipment was $3.5 \mathrm{kV}$. The measurement was carried out at temperature $T=1.5 \mathrm{~K}$ in the $E$-field cooling condition; the system was set to $10 \mathrm{~K}, E$ was applied, and then the system was cooled down to $1.5 \mathrm{~K}$.

\section{RESULTS AND ANALYSES}

To directly confirm the magnetic structure having the easy axis along $\langle 100\rangle$ directions, which was determined by the magnetization measurements [29], the polarized-neutron measurement was carried on a $\mathrm{Ba}_{2} \mathrm{CoGe}_{2} \mathrm{O}_{7}$ single crystal at zero electric field. We estimate the direction of the magnetic moment and the ratio of the magnetic domain simultaneously from the angle dependence of the magnetic intensities at the equivalent $\boldsymbol{Q}$ points in the $c$ plane. The intensities of the magnetic Bragg reflections were measured at the equivalent $\boldsymbol{Q}$ points shown in Fig. 2(a). The average intensity at the equivalent $\boldsymbol{Q}$ points to $\boldsymbol{Q}=(1,2,0)$ is normalized to 1.0 , and the intensity at each $\boldsymbol{Q}$ point is plotted as a function of the angle between the scattering vector $Q$ and the $a$ axis by the opened circles in Fig. 2(b). This result shows that the intensities have angle dependence in the $c$ plane.

In the neutron scattering experiment only the magnetic moment perpendicular to the scattering vector $\boldsymbol{Q}$ contributes to the magnetic intensity. Here we consider the angle dependence of the magnetic intensities at the equivalent $\boldsymbol{Q}$ points. As the average intensity at the equivalent $\boldsymbol{Q}$ points is normalized to 1.0 , the magnetic intensity in the collinear structure is expressed as $2\left(1-S_{Q}^{2} / S^{2}\right)$. The $S_{Q}$ is the component of the magnetic moment parallel to the scattering vector $\boldsymbol{Q}$. The term $2\left(1-S_{Q}^{2} / S^{2}\right)$ in the collinear structure does not depend on the structure factor, and it is determined only by the direction of the magnetic moment. Calculated intensities of collinear-antiferromagnetic structure with the spins aligned along [100] and [010] directions are indicated by the dashed and dotted lines, respectively, in Fig. 2(b). The observed angle dependence of the intensities means the imbalance of the magnetic domains. The volume ratios of the magnetic domains with the easy axis along [100] and [010] directions are estimated to be 57 and $43 \%$, respectively, as indicated by the red curve in Fig. 2(b). The intensities at the $Q$ points $(1,0,0)$ and $(0,1,0)$ are also plotted by closed circles in Fig. 2(b). Here the average intensity is normalized to 1.0. The intensities at the $\boldsymbol{Q}$ points $(1,0,0)$ and $(0,1,0)$ coincide with the angle dependence of the intensities at the $\boldsymbol{Q}$ points which are equivalent to $\boldsymbol{Q}=(1,2,0)$. In contrast, the observed angle dependence of the intensities can not be explained by the magnetic structure having the easy axis along $\langle 110\rangle$ directions, of which the intensity curve shifts by $45^{\circ}$. The fitting result that the volumes of the magnetic domains with the easy axis along [110] and [110] directions are almost equal can not reproduce the observed angle dependence as shown by blue curve in Fig. 2(b). These results are consistent with the magnetic structure having the easy axis along $\langle 100\rangle$ directions.

To test the possibility of the continuous control of the magnetic moment by $E$, the intensities of the magnetic Bragg reflections at the $Q$ points shown in Fig. 3(a) were measured under $E$. As shown in Fig. 3(b) the intensities show linear changes with increasing $E$. Here the intensities at $E=0$ are normalized to unity to compare the change ratio of the intensities. As $E$ increases, the intensities at the $Q$ points with 
(a)
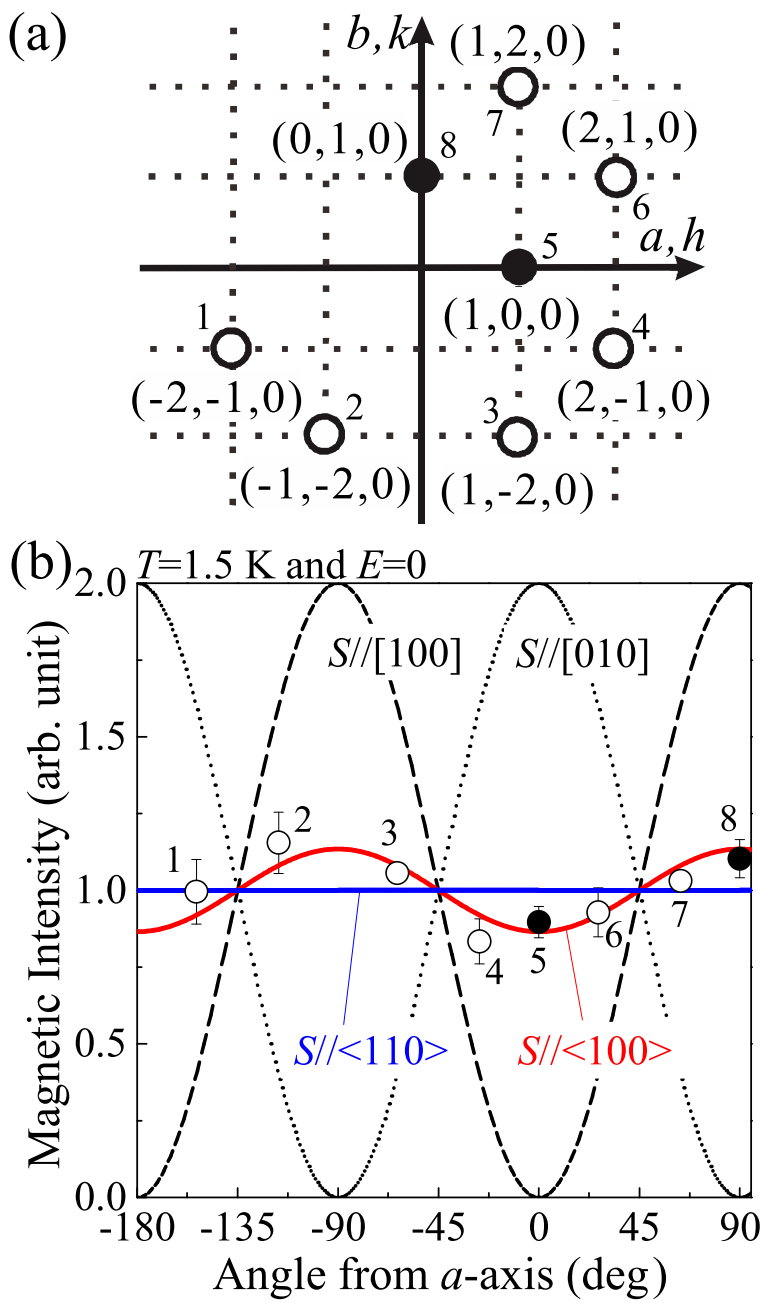

FIG. 2. (a) Two kinds of equivalent $Q$ points measured at $T=$ $1.5 \mathrm{~K}$. (b) Magnetic intensities measured at $T=1.5 \mathrm{~K}$ without $E$ against the angle from the $a$ axis. Opened circles exhibit the $\boldsymbol{Q}$ points which are equivalent to $(1,2,0)$. Closed circles exhibit the $\boldsymbol{Q}$ points which are equivalent to $(1,0,0)$. Dashed and dotted lines show the angle dependence of the magnetic intensities for the magnetic structures having the magnetic moments along [100] and [010], respectively. Red line shows the angle dependence of the magnetic intensity obtained by the fitting for the magnetic domains with the easy axis along [100] and [010] directions. Blue line shows the angle dependence of the magnetic intensity obtained by the fitting for the magnetic domains with the easy axis along [110] and [110] directions.

$h>0$ and $k>0$ decrease. In contrast at the $Q$ points with $h>$ 0 and $k<0$, the intensities increase with the increase of $E$. The trend of the increase and decrease of the intensities reverses as $E$ reverses. The magnetic intensities under $E$ are replotted as a function of the angle between the scattering vector $\boldsymbol{Q}$ and the $a$ axis in Fig. 3(c). This shows that the intensities have the sinusoidal modulation in the $c$ plane and that the amplitude increases with $E$. The changes of the magnetic intensities correspond to the change of the spin arrangement as we will see below.

To consider how the arrangement of the magnetic moment changes with $E$, we made three assumptions. The (a)

(b)
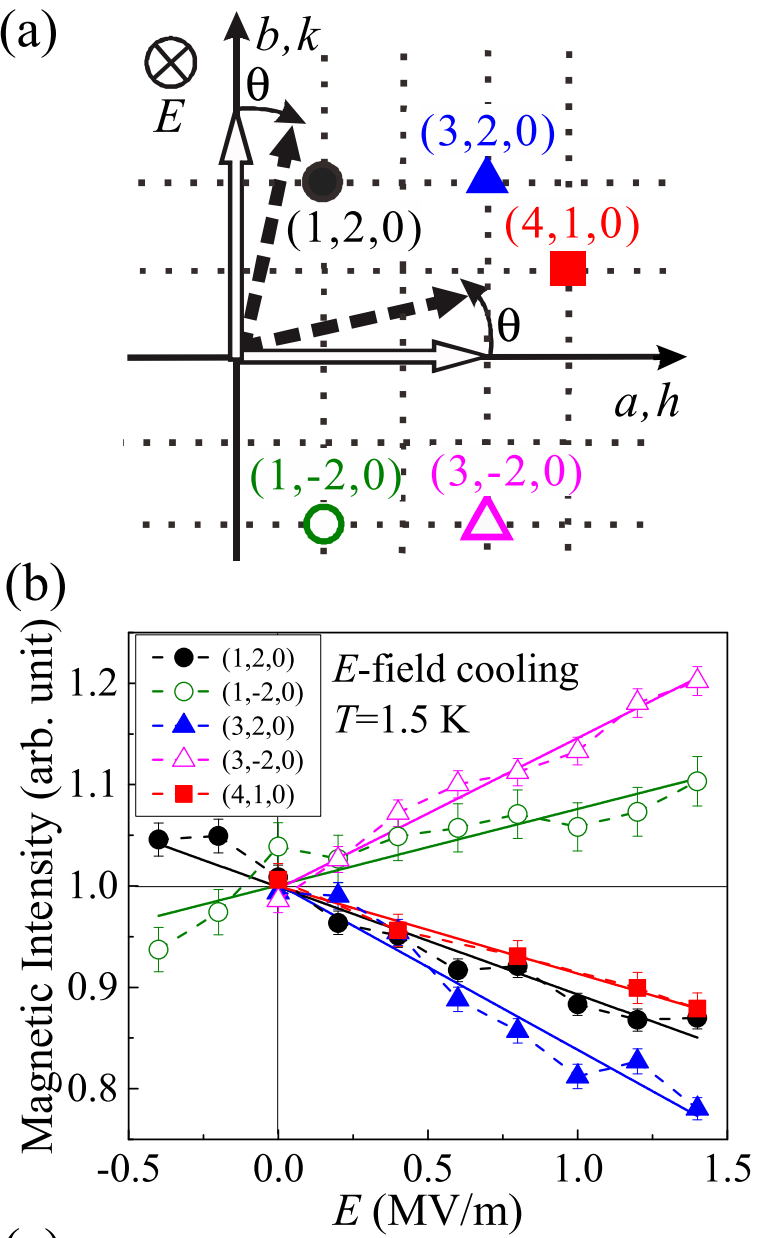

(c)

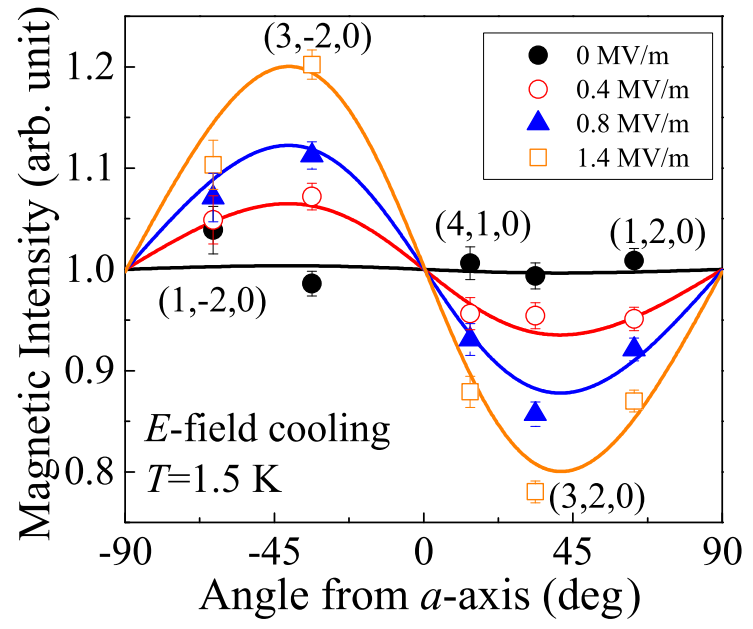

FIG. 3. (a) Relationship between the measured $Q$ points and the magnetic moments. The arrows exhibit the direction of the magnetic moments. (b) $E$ dependence of the magnetic intensities at several $\boldsymbol{Q}$ points. The measurement was carried out at $1.5 \mathrm{~K}$ after $E$-field cooling. (c) Magnetic intensities measured at $1.5 \mathrm{~K}$ against the angle from the $a$ axis under several $E$-field conditions. Solid line shows the results fitted by the rotation angle of the magnetic moments.

first one is that the collinear-antiferromagnetic structure is preserved by the large antiferromagnetic interaction between the nearest-neighbor spins $[26,29,33]$. No observed change 


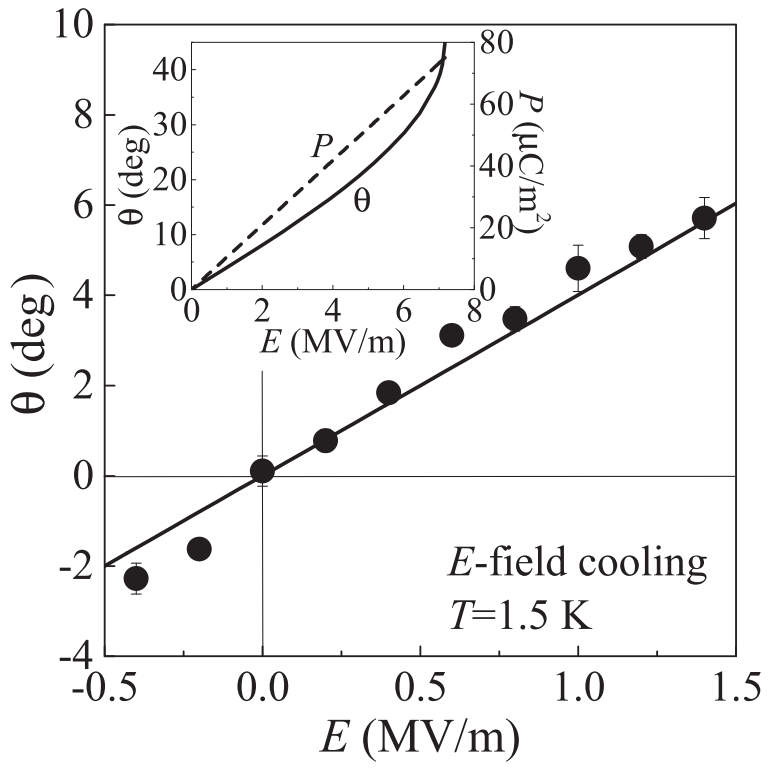

FIG. 4. $E$ dependence of the rotation angle of the magnetic moments from $a$ and $b$ axes. Solid line shows the rotation angle estimated by the energy of the spin-nematic interaction. Inset shows the rotation angle and the electric polarization calculated in the wide- $E$ region.

of the propagation vector under $E$ supports this assumption. The second one is that the $\boldsymbol{p}$ direction does not deviate from the $c$ axis. The large single ion anisotropy [29] leads to the constraint that the magnetic moment is confined in the $c$ plane and the $\boldsymbol{p}$ direction is fixed to the $c$ axis from the relationship of Eq. (1). In the second assumption the decrease of the magnetic intensities at the first quadrant of the ( $h k 0)$ plane means that the direction of the magnetic moment shifts from [100] or [010] to somewhere in the first quadrant of the $a-b$ plane as indicated by dashed arrows in Fig. 3(a). The third assumption is that the angle between the magnetic moment and the $a$ axis in one domain and that between the magnetic moment and the $b$ axis in the other domain is the same. From the change in the ratio of the magnetic intensities we estimated the rotation angle of the magnetic moments. The normalized magnetic intensities as a function of the angle between the $Q$ point and $a$ axis for several $E$ 's are shown in Fig. 3(c). The solid curve is the fitting result using our assumed model. Here the fitting parameter is only the rotation angle of the magnetic moment from the $a$ and $b$ axes. The obtained rotation angles are plotted as a function of $E$ in Fig. 4. With increasing $E$ the magnetic moment rotates to the direction along [110] with the rotation angle away from the $a$ and $b$ axes showing a linear behavior. As $E$ is reversed, the magnetic moment rotates in the opposite direction towards [110]. Thus, the magnetic moment direction is controlled directly and continuously by $E$.

\section{DISCUSSION}

We discuss the $E$ dependence of the local-spin direction in the framework of the effective-low-energy Hamiltonian where the spins forming the collinear-antiferromagnetic structure are confined in the $a-b$ plane and the electric polarizations are confined along the $c$ axis. The mean-field energy of two sites is

$$
\begin{aligned}
E_{\mathrm{MF}}(\theta)= & -4 J_{p} p_{Z}^{(A)} p_{Z}^{(B)}-E\left(p_{Z}^{(A)}+p_{Z}^{(B)}\right) \\
= & -4 J_{p}^{\mathrm{eff}}\left\langle O_{X Y}^{(A)}\right\rangle\left\langle O_{X Y}^{(B)}\right\rangle \\
& -E K_{c}\left(\left\langle O_{X Y}^{(A)}\right\rangle+\left\langle O_{X Y}^{(B)}\right\rangle\right),
\end{aligned}
$$

where $\left\langle O_{X Y}^{(A)}\right\rangle=\langle O\rangle \sin (2 \theta-2 \kappa)$ and $\left\langle O_{X Y}^{(B)}\right\rangle=\langle O\rangle \sin (2 \theta+$ $2 \kappa) . \theta$ is the rotation angle of the magnetic moment from the $a$ and $b$ axes. $\kappa$ is the tilt angle of $\mathrm{CoO}_{4}$ from $\langle 100\rangle$, as shown in Fig. 1(a). The sign of $\left\langle O_{X Y}\right\rangle$ depends on $\theta$ and $\kappa$, as shown by the plus and minus signs in Fig. 1. In mean-field approximation the self-consistent value of $\langle O\rangle$ is estimated to be 0.911 from the antiferromagnetic exchange interaction $J_{\perp}=0.208 \mathrm{meV}$ and the single ion anisotropy $D=1.034 \mathrm{meV}$ (see Appendix) [29]. In order to calculate the minimum energy in Eq. (4), we need to estimate $p_{Z}\left(\theta=45^{\circ}\right)=K_{c}\langle O\rangle \cos (2 \kappa)$ from the $H$ dependence of the electric polarization along the $c$ axis $\left(P_{c}\right)$ reported by Murakawa et al. [27]. By applying $H$ along [110], the magnetic moments flop from $\langle 100\rangle$ to [110], and the antiferroelectric state changes to the ferroelectric one. In Ref. [27], an inflection point of the $P_{c}$ curve as a function of $H$ was observed at about $0.5 \mathrm{~T}$, and we consider that the inflection point corresponds with the transition from the antiferroelectric to the ferroelectric states. We assume that the direction of the magnetic moment is along [110] at the inflection point, $75 \mu \mathrm{C} / \mathrm{m}^{2}$. Then, $p_{Z}\left(45^{\circ}\right)$ is estimated to be $1.47 \times 10^{-26} \mu \mathrm{C} \mathrm{m}$. From $J_{p}^{\text {eff }}=-0.198 \mu \mathrm{eV}$ [29], $\langle O\rangle=$ 0.911 , and $p_{Z}\left(45^{\circ}\right)=1.47 \times 10^{-26} \mu \mathrm{Cm}$, we calculated the minimum energy in Eq. (4). Then, we obtained the relationship between $E$ and $\theta$, as indicated by the solid line in Fig. 4 (see Appendix). The observed $E$ dependence of the rotation angle is consistent with the value estimated from the dielectric energy and the spin-nematic interaction. Furthermore, we found that $E_{\text {sat }}=7.17 \mathrm{MV} / \mathrm{m}$ needs to be applied in order to realize the magnetic structure having the magnetic moments along the [110] direction, as shown in the inset of Fig. 4. The $E$ dependence of $\theta$ depends on $J_{p}^{\text {eff }}$ and the smaller value of $\left|J_{p}^{\text {eff }}\right|$ gives smaller $E_{\text {sat }}$.

The $E$ dependence of the electric polarization calculated from the $\theta$ dependence of $p_{Z}$ and the $E$ dependence of $\theta$ is shown in the inset of Fig. 4 (see Appendix). The estimated polarization shows a linear behavior with $E$. This is different from the typical antiferroelectric system where the first order transition from the antiferroelectric to the ferroelectric states is observed with $E[34,35]$. Since the local polarization of $\mathrm{CoO}_{4}$ tetrahedron has the continuous change through the rotation of the magnetic moments, the dielectric change from the antiferroelectric to the ferroelectric states would occur gradually with increasing $E$, as shown in Figs. 1(b) to 1(e). Both continuous changes of the dielectric state and the spin direction with $E$ are the unique characteristics of $\mathrm{Ba}_{2} \mathrm{CoGe}_{2} \mathrm{O}_{7}$. In the spin-dependent $d$ - $p$ hybridization mechanism, furthermore, the multiferroics having the antiferroelectric ground state is expected to exhibit the same continuous changes with $E$. 


\section{CONCLUSION}

We demonstrated that the direction of the local magnetic moment in $\mathrm{Ba}_{2} \mathrm{CoGe}_{2} \mathrm{O}_{7}$ is controlled continuously by the electric field. The rotation angle of the magnetic moment is explained quantitatively by the Hamiltonian including the spin-nematic interaction. Continuous changes of both spin and electric polarization simultaneously by the applied electric field are very unique. Our finding will open a door to an alternative electric device.

\section{ACKNOWLEDGMENTS}

The neutron experiment was performed by using TASP at SINQ/PSI, Switzerland, which was transferred from 5G(PONTA) with the approval of Institute for Solid State Physics, the University of Tokyo (Proposal No. 13532) and the Japan Atomic Energy Agency, Tokai, Japan. This work was supported by KAKENHI (24340077, 24740224, 15K05123, and 26400332).

\section{APPENDIX: CALCULATION DETAILS}

We first discuss the mean-field solution of the spin system. The mean-field Hamiltonian for the spin system is expressed as

$$
\mathcal{H}_{\mathrm{MF}}=D\left(S^{Z}\right)^{2}-h\left(\cos \phi S^{X}+\sin \phi S^{Y}\right) .
$$

Here, $S^{\alpha}$ represents the $\alpha(=X, Y, Z)$ component of the $S=3 / 2$ spin operator. $h$ represents an effective molecular magnetic field coming from the nearest neighbor sites. We discuss $h$ later. $\phi$ represents the angle of the molecular field measured from the $X$ axis. $D$ and $J_{\perp}$ represent the easy-plane single-ion anisotropy and the $X Y$ component of the intersite exchange interaction, respectively. They were extracted as $D=1.034 \mathrm{meV}$ and $J_{\perp}=0.208 \mathrm{meV}$ from the magnon dispersion relations obtained by the inelastic neutron scattering measurements [29]. Under the strong easy-plane anisotropy, we note that the spin direction is confined in the $X-Y$ plane. The weak anisotropic terms in the Hamiltonian, DzyaloshinskiiMoriya interaction, and the spin-nematic interaction [29], can be neglected when we consider the expectation value of the spin-nematic operator. The ground state of Eq. (A1) is given by

$$
\begin{aligned}
|\mathrm{GS}\rangle= & \frac{1}{N_{0}}\left[e^{-i \frac{3}{2} \phi} \sqrt{3} h\left|\frac{3}{2}\right\rangle+e^{-i \frac{1}{2} \phi} f(D, h)\left|\frac{1}{2}\right\rangle\right. \\
& \left.+e^{i \frac{1}{2} \phi} f(D, h)\left|-\frac{1}{2}\right\rangle+e^{i \frac{3}{2} \phi} \sqrt{3} h\left|-\frac{3}{2}\right\rangle\right]
\end{aligned}
$$

with

$$
\begin{aligned}
f(D, h) & =2 D+h+2 \sqrt{D^{2}+D h+h^{2}}, \\
N_{0} & =\sqrt{6 h^{2}+2 f^{2}(D, h)} .
\end{aligned}
$$

Here, $|m\rangle\left(m=\frac{3}{2}, \frac{1}{2},-\frac{1}{2},-\frac{3}{2}\right)$ represents the wave function for the $S^{Z}=m$ state. The expectation values of the spin operators are calculated as

$$
\left\langle S_{X}\right\rangle=\langle S\rangle \cos \phi, \quad\left\langle S_{Y}\right\rangle=\langle S\rangle \sin \phi,
$$

with

$$
\langle S\rangle=\frac{D+2 h+\sqrt{D^{2}+D h+h^{2}}}{2 \sqrt{D^{2}+D h+h^{2}}} .
$$

The effective molecular field is defined by

$$
h=4 J_{\perp}\langle S\rangle .
$$

We can determine the value of $\langle S\rangle$ by solving Eq. (A5) self-consistently with Eq. (A6). We obtain $\langle S\rangle=1.381$ for $D=1.034 \mathrm{meV}$ and $J_{\perp}=0.208 \mathrm{meV}$. This fixes the value of the molecular field as $h=1.149 \mathrm{meV}$ via Eq. (A6). The expectation value of the spin-nematic operator, $O_{X Y}=$ $S^{X} S^{Y}+S^{Y} S^{X}$, is calculated as

$$
\left\langle O_{X Y}\right\rangle=\langle O\rangle \sin (2 \phi),
$$

where $\langle O\rangle$ is given by

$$
\langle O\rangle=\frac{3 h}{2 \sqrt{D^{2}+D h+h^{2}}} .
$$

We obtain $\langle O\rangle=0.911$ for the fixed $h$ and $D$.

Next, we study the electric polarization under a uniform electric field $E$ applied parallel to the $c$ axis. In this case, the weak spin-nematic interaction and the electric field determine the angle of the ordered magnetic moment. The mean-field energy for the electric polarizations per two spin sites (A and B sites) is expressed as

$$
E_{\mathrm{MF}}(\theta)=-4 J_{p}^{\mathrm{eff}}\left\langle O_{X Y}^{(A)}\right\rangle\left\langle O_{X Y}^{(B)}\right\rangle-E\left(p_{Z}^{(A)}+p_{Z}^{(B)}\right) .
$$

Here, $\left\langle O_{X Y}^{(A)}\right\rangle$ and $\left\langle O_{X Y}^{(B)}\right\rangle$ are expectation values of the spinnematic operators at the $\mathrm{A}$ and $\mathrm{B}$ sites, respectively. $J_{p}^{\text {eff }}$ represents the effective interaction between the spin-nematic operators. It was estimated as $J_{p}^{\text {eff }}=-0.198 \mu \mathrm{eV}$ [29]. The expectation values of the spin-nematic operators are expressed as

$$
\begin{aligned}
& \left\langle O_{X Y}^{(A)}\right\rangle=\langle O\rangle \sin (2 \theta-2 \kappa), \\
& \left\langle O_{X Y}^{(B)}\right\rangle=\langle O\rangle \sin (2 \theta+2 \kappa),
\end{aligned}
$$

where $\theta$ represents the angle of the magnetic moment measured from the $a$ and $b$ axes. $\kappa$ is the tilt angle of $\mathrm{CoO}_{4}$ from $\langle 100\rangle$. From the above discussion, the value of $\langle O\rangle$ is already fixed. In Eq. (A9), $p_{Z}^{(A)}$ and $p_{Z}^{(A)}$ represent the local electric polarizations at the $A$ and $B$ sites, respectively. They are expressed by the spin-nematic operators as

$$
\begin{aligned}
& p_{Z}^{(A)}=K_{c}\left\langle O_{X Y}^{(A)}\right\rangle=K_{c}\langle O\rangle \sin (2 \theta-2 \kappa), \\
& p_{Z}^{(B)}=K_{c}\left\langle O_{X Y}^{(B)}\right\rangle=K_{c}\langle O\rangle \sin (2 \theta+2 \kappa) .
\end{aligned}
$$

Here, $K_{c}$ is a constant connecting the spin-nematic and the electric polarization.

For later convenience, we introduce the following averaged value of the electric polarization per one spin site:

$$
p_{\text {Zav }}(\theta)=\frac{1}{2} K_{c}\left[\left\langle O_{X Y}^{(A)}\right\rangle+\left\langle O_{X Y}^{(B)}\right\rangle\right]=p_{Z \mathrm{Zav}}\left(45^{\circ}\right) \sin (2 \theta) .
$$

Here, we defined $p_{Z a v}\left(45^{\circ}\right)$ as

$$
p_{\text {Zav }}\left(45^{\circ}\right)=K_{c}\langle O\rangle \cos (2 \kappa)
$$


for $\theta=45^{\circ}$. In Eq. (A12), we can see that $p_{\text {Zav }}(\theta)$ takes the maximum value at $\theta=45^{\circ}$. Therefore, it is expected that the magnetic moments are aligned along the [110] direction under strong electric fields applied along the $c$ axis. The local electric polarizations at the $\mathrm{A}$ and $\mathrm{B}$ sites are also expressed as

$$
\begin{aligned}
& p_{Z}^{(A)}=\frac{p_{Z \mathrm{av}}\left(45^{\circ}\right)}{\cos (2 \kappa)} \sin (2 \theta-2 \kappa), \\
& p_{Z}^{(B)}=\frac{p_{\mathrm{Zav}}\left(45^{\circ}\right)}{\cos (2 \kappa)} \sin (2 \theta+2 \kappa) .
\end{aligned}
$$

The averaged value of these polarizations, i.e., Eq. (A12), is observable by macroscopic measurements.

Next, we determine the angle of the magnetic moment under the electric field. Substituting Eqs. (A10) and (A14) into Eq. (A9), we obtain

$$
E_{\mathrm{MF}}(\theta)=-a \sin (2 \theta)-b \cos (4 \theta)+c,
$$

with

$$
\begin{aligned}
& a=2 p_{Z \mathrm{av}}\left(45^{\circ}\right) E, \\
& b=-2 J_{p}^{\mathrm{eff}}\langle O\rangle^{2}, \\
& c=-2 J_{p}^{\mathrm{eff}}\langle O\rangle^{2} \cos (4 \kappa) .
\end{aligned}
$$

For later convenience, we rewrite

$$
\frac{a}{b}=\frac{4 E}{E_{\mathrm{sat}}},
$$

where we introduced

$$
E_{\mathrm{sat}}=\frac{-4 J_{p}^{\mathrm{eff}}\langle O\rangle^{2}}{p_{\mathrm{Zav}}\left(45^{\circ}\right)} .
$$

$E_{\mathrm{MF}}(\theta)$ is then expressed as

$$
\begin{aligned}
E_{\mathrm{MF}}(\theta) & =-b\left[\frac{4 E}{E_{\mathrm{sat}}} \sin (2 \theta)+\cos (4 \theta)\right]+c \\
& =-b\left[\frac{4 E}{E_{\mathrm{sat}}} \sin (2 \theta)-2 \sin ^{2}(2 \theta)\right]-b+c \\
& =2 b\left[\sin (2 \theta)-\frac{E}{E_{\mathrm{sat}}}\right]^{2}-b\left[1+\left(\frac{E}{E_{\mathrm{sat}}}\right)^{2}\right]+c .
\end{aligned}
$$

The angle of the magnetic moment under the electric field is determined to minimize $E_{\mathrm{MF}}(\theta)$. For the antiferroelectric interaction $\left(J_{p}^{\text {eff }}<0\right), b$ is positive. Therefore, $E_{\mathrm{MF}}(\theta)$ is minimized by $\sin (2 \theta)=E / E_{\text {sat }}$. For $\left|E / E_{\text {sat }}\right|>1, \quad \theta=(\pi / 4) \operatorname{sgn}(E)$ minimizes $E_{\mathrm{MF}}(\theta)$. Thus, the result is summarized as

$$
\theta(E)= \begin{cases}\frac{1}{2} \sin ^{-1}\left(\frac{E}{E_{\mathrm{sat}}}\right) & \text { for }|E| \leqslant E_{\mathrm{sat}} \\ \frac{\pi}{4} \operatorname{sgn}(E) & \text { for }|E| \geqslant E_{\text {sat }} .\end{cases}
$$

Now we can understand that $E_{\text {sat }}$ represents the saturation electric field above which $|\theta|$ becomes $\pi / 4$ (the magnetic moment is aligned along the [110] or [110] directions) and the electric polarization saturates. Since the $\theta(E)$ given by Eq. (A20) is an odd function of $E$, the angle direction of the moment reverses with $E \rightarrow-E$. For $|E| \ll E_{\text {sat }}$, Eq. (A20) is approximated as

$$
\theta(E)=\frac{E}{2 E_{\mathrm{sat}}}
$$

Therefore, $1 /\left(2 E_{\text {sat }}\right)$ represents the slope of $\theta(E)$. The $E$ dependence of the electric polarization is calculated as

$$
p_{\text {Zav }}(E)=p_{\text {Zav }}\left(45^{\circ}\right) \sin (2 \theta)=p_{\text {Zav }}\left(45^{\circ}\right) \frac{E}{E_{\mathrm{sat}}} .
$$

Here, we used Eq. (A20) for $\sin (2 \theta)=E / E_{\text {sat }}$. Equation (A22) indicates that the electric polarization linearly increases with $E$ up to the saturation field. For $|E|>E_{\text {sat }}$, the electric polarization saturates and takes the value of $p_{\mathrm{Zav}}\left(45^{\circ}\right)$ $\operatorname{sgn}(E)$.

As in Eqs. (A20) and (A22), $\theta(E)$ and $p_{\text {Zav }}(E)$ are functions of $E / E_{\text {sat }}$. The saturation electric field is defined by Eq. (A18). We know the value of $J_{p}^{\text {eff }}$ and $\langle O\rangle$ [29], while $p_{Z \text { av }}\left(45^{\circ}\right)$ can be estimated from the magnetic field-induced electric polarization reported by Murakawa and coworkers [27]. Therefore, $E_{\text {sat }}$ can be fixed and we can plot $\theta(E)$ and $p_{Z \text { av }}(E)$ for $\mathrm{Ba}_{2} \mathrm{CoGe}_{2} \mathrm{O}_{7}$.
[1] M. Fiebig, J. Phys. D: Appl. Phys. 38, R123 (2005).

[2] W. Eerenstein, N. D. Mathur, and J. F. Scott, Nature (London) 442, 759 (2006)

[3] T. Kimura, T. Goto, H. Shintani, K. Ishizaka, T. Arima, and Y. Tokura, Nature (London) 426, 55 (2003).

[4] D. N. Astrov, Sov. Phys. JETP 11, 708 (1960).

[5] V. J. Folen, G. T. Rado, and E. W. Stalder, Phys. Rev. Lett. 6, 607 (1961)

[6] A. Iyama and T. Kimura, Phys. Rev. B 87, 180408(R) (2013).

[7] W. F. Brown, Jr., R. M. Hornreich, and S. Shtrikman, Phys. Rev. 168, 574 (1968)

[8] T. Goto, T. Kimura, G. Lawes, A. P. Ramirez, and Y. Tokura, Phys. Rev. Lett. 92, 257201 (2004).
[9] M. Soda, T. Ishikura, H. Nakamura, Y. Wakabayashi, and T. Kimura, Phys. Rev. Lett. 106, 087201 (2011).

[10] K. Kimura, H. Nakamura, K. Ohgushi, and T. Kimura, Phys. Rev. B 78, 140401(R) (2008).

[11] H. Katsura, N. Nagaosa, and A. V. Balatsky, Phys. Rev. Lett. 95, 057205 (2005).

[12] M. Mostovoy, Phys. Rev. Lett. 96, 067601 (2006).

[13] C. Jia, S. Onoda, N. Nagaosa, and J. H. Han, Phys. Rev. B 76, 144424 (2007).

[14] T. Arima, J. Phys. Soc. Jpn. 76, 073702 (2007).

[15] Y. Tokunaga, Y. Taguchi, T. Arima, and Y. Tokura, Nat. Phys. 8, 838 (2012). 
[16] Y. Chai, S. Kwon, S. Chun, I. Kim, B. Jeon, K. Kim, and S. Lee, Nat. Comm. 5, 4208 (2014).

[17] E. Ascher, H. Rieder, H. Schmid, and H. Stössel, J. Appl. Phys. 37, 1404 (1966).

[18] M. Saito, K. Ishikawa, S. Konno, K. Taniguchi, and T. Arima, Nat. Mater. 8, 634 (2009).

[19] Y. Yamasaki, H. Sagayama, T. Goto, M. Matsuura, K. Hirota, T. Arima, and Y. Tokura, Phys. Rev. Lett. 98, 147204 (2007).

[20] I. Cabrera, M. Kenzelmann, G. Lawes, Y. Chen, W. C. Chen, R. Erwin, T. R. Gentile, J. B. Leao, J. W. Lynn, N. Rogado, R. J. Cava, and C. Broholm, Phys. Rev. Lett. 103, 087201 (2009).

[21] M. Soda, K. Kimura, T. Kimura, M. Matsuura, and K. Hirota, J. Phys. Soc. Jpn. 78, 124703 (2009).

[22] T. Lottermoser, T. Lonkai, U. Amann, D. Hohlwein, J. Ihringer, and M. Fiebig, Nature (London) 430, 541 (2004).

[23] Y. Hiraoka, Y. Tanaka, M. Oura, Y. Wakabayashi, and T. Kimura, J. Magn. Magn. Mater. 384, 160 (2015).

[24] J. S. White, K. Prša, P. Huang, A. A. Omrani, I. Živković, M. Bartkowiak, H. Berger, A. Magrez, J. L. Gavilano, G. Nagy, J. Zang, and H. M. Rønnow, Phys. Rev. Lett. 113, 107203 (2014).

[25] T. Sato, T. Masuda, and K. Uchinokura, Physica B 329-333, 880 (2003).
[26] A. Zheludev, T. Sato, T. Masuda, K. Uchinokura, G. Shirane, and B. Roessli, Phys. Rev. B 68, 024428 (2003).

[27] H. Murakawa, Y. Onose, S. Miyahara, N. Furukawa, and Y. Tokura, Phys. Rev. Lett. 105, 137202 (2010).

[28] H. T. Yi, Y. J. Choi, S. Lee, and S.-W. Cheong, Appl. Phys. Lett. 92, 212904 (2008).

[29] M. Soda, M. Matsumoto, M. Månsson, S. Ohira-Kawamura, K. Nakajima, R. Shiina, and T. Masuda, Phys. Rev. Lett. 112, 127205 (2014).

[30] J. Romhányi, M. Lajkó, and K. Penc, Phys. Rev. B 84, 224419 (2011).

[31] S. Miyahara and N. Furukawa, J. Phys. Soc. Jpn. 80, 073708 (2011).

[32] M. Janoschek, S. Klimko, R. Gaehler, B. Roessli, and P. Böni, Physica B 397, 125 (2007).

[33] Although $\mathcal{H}_{D M}$ and $\mathcal{H}_{p}$ can give rise to a noncollinear spin structure, the coupling parameters in these interactions are estimated to be much smaller than the predominant exchange interaction $\mathcal{H}_{x x z}$.

[34] G. Shirane, E. Sawaguchi, and Y. Takagi, Phys. Rev. 84, 476 (1951).

[35] S. Horiuchi, F. Kagawa, K. Hatahara, K. Kobayashi, R. Kumai, Y. Murakami, and Y. Tokura, Nat. Commun. 3, 1308 (2012). 